\title{
The effect of dietary phosphatidylcholine supplementation on lipid profile in mild hyperlipidaemic individuals
}

\author{
M. Jan, P.S. Thondre, A. El-Chab and H.J. Lightowler \\ Functional Food Centre, Oxford Brookes University, Oxford OX3 OBP, UK
}

Hyperlipidaemia is a risk factor for cardiovascular disease and, according to the British Heart Foundation (2015), over half of England's adult population suffers from high cholesterol levels ${ }^{(1)}$. Phosphatidylcholine (PC) is a class of lipids mainly found in egg yolk and soy bean. Several studies have suggested that PC supplementation can help in improving blood lipid profile ${ }^{(2-3)}$. The aim of the present study was to investigate the effect of two different doses of PC supplementation (600 mg and $2400 \mathrm{mg}$ ) on blood lipid profile in individuals with mild elevated levels of low-density lipoprotein cholesterol (LDL-C).

In a parallel intervention study, ten participants (mean age $44 \pm 13$ years) were randomly allocated to either a low dose (600 mg; LDG) or high dose (2400 mg; HDG) PC supplement group for 4 weeks. A capillary blood sample was taken at baseline, after two weeks and four weeks of PC supplementation. Measurement of total cholesterol (TC), LDL-C, high-density lipoprotein cholesterol (HDL-C), non-HDL-C, TC/HDL-C ratio and triglycerides (TG) was analysed using the CardioChek Professional Analyser.

At baseline, the mean blood lipid concentrations were similar in both groups. After four weeks of treatment, there was a significant $(P<0.05)$ reduction in TC in the HDG compared to the LDG. Levels of LDL-C, non-HDL-C and TC/HDL ratio show a slight decrease at week 4 in both groups (Table 1).

Table 1. Comparison of the change in blood lipid profile from baseline between the two groups

\begin{tabular}{|c|c|c|c|}
\hline Variables & LDG $n=6$ & HDG $n=4$ & $P$ Value \\
\hline \multicolumn{4}{|c|}{ Total cholesterol (mmol/l) } \\
\hline 2weeks & $-0 \cdot 2 \pm 0 \cdot 7$ & $-0 \cdot 5 \pm 0 \cdot 8$ & $0 \cdot 476$ \\
\hline 4 weeks & $0 \cdot 0 \pm 0 \cdot 3$ & $-0.5 \pm 0.4$ & 0.035 \\
\hline \multicolumn{4}{|c|}{ TGs (mmol/l) } \\
\hline 2 weeks & $0 \cdot 2 \pm 0 \cdot 3$ & $-0 \cdot 0 \pm 0.8$ & 0.476 \\
\hline 4 weeks & $0 \cdot 3 \pm 0 \cdot 7$ & $-0.6 \pm 0.8$ & $0 \cdot 171$ \\
\hline \multicolumn{4}{|c|}{$H D L-C$ (mmolll) } \\
\hline 2 weeks & $0 \cdot 1 \pm 0 \cdot 4$ & $-0 \cdot 0 \pm 0 \cdot 1$ & 0.499 \\
\hline 4 weeks & $0 \cdot 2 \pm 0 \cdot 3$ & $-0 \cdot 1 \pm 0 \cdot 2$ & $0 \cdot 110$ \\
\hline \multicolumn{4}{|c|}{$L D L-C$ (mmolll) } \\
\hline 2weeks & $-0.4 \pm 0 \cdot 3$ & $-0.5 \pm 0.7$ & $0 \cdot 803$ \\
\hline 4weeks & $-0 \cdot 3 \pm 0 \cdot 5$ & $-0 \cdot 2 \pm 0.7$ & $0 \cdot 706$ \\
\hline \multicolumn{4}{|c|}{ Non-HDL-C (mmolll) } \\
\hline 2 weeks & $-0 \cdot 3 \pm 0 \cdot 3$ & $-0.5 \pm 0.9$ & $0 \cdot 684$ \\
\hline 4 weeks & $-0 \cdot 2 \pm 0 \cdot 3$ & $-0.4 \pm 0.4$ & $0 \cdot 323$ \\
\hline \multicolumn{4}{|c|}{ TC/HDL ratio (mmolll) } \\
\hline 2 weeks & $-0 \cdot 3 \pm 0 \cdot 3$ & $-0.4 \pm 0.9$ & 0.934 \\
\hline 4 weeks & $-0 \cdot 3 \pm 0.4$ & $-0.3 \pm 0.7$ & $0 \cdot 850$ \\
\hline
\end{tabular}

The results from the current study indicate that $2400 \mathrm{mg}$ of PC supplementation can significantly decrease TC levels and may help reduce LDL-C levels. LDL-C is considered to be a primary indicator for developing CVD. Although many hypolipidaemic drugs are available for patients, these may not fully achieve the required levels of lipids and may cause adverse effects such as increasing the risk of developing diabetes mellitus. Further research in this area is required to confirm the effect of PC supplementation on lipid profile in patients at risk of developing CVD.

1. British Heart Foundation (2015) Cardiovascular Disease Statistics Factsheet. Available at: https://www.bhf.org.uk/research/heart-statistics (Accessed: 3 April 2017).

2. Childs M, Bowlin J et al. (1981) Atherosclerosis 1-2, 217-228.

3. Høie L, Morgenstern E et al. (2005) Eur J Nutr 2, 65-71. 